\title{
Visual working memory is enhanced by mixed strategy use and semantic coding.
}

\author{
Louise A. Brown, and Robert W. Wesley \\ Division of Psychology, Nottingham Trent University
}

To cite:

Brown, L.A., \& Wesley, R. (2013). Visual working memory is enhanced by mixed strategy use and semantic coding. Journal of Cognitive Psychology, 25 (3), 328-338.

DOI:10.1080/20445911.2013.773004 


\begin{abstract}
Visual working memory is enhanced by processes related to verbalisation. However, the mechanism underlying this enhancement is unclear. Experiment 1 investigated the potential contribution of the phonological loop of working memory, by assessing the effects of articulatory suppression on two versions of the Visual Patterns Test - one low and one high in availability of verbal coding. The lack of interaction suggested that the phonological loop is not responsible; however, active use of combined verbal and visual strategies, as well as activated semantic knowledge, both appear to be related to increased capacity. Experiment 2 assessed the role of central executive resources. Because central executive suppression removed the benefit of the high verbal coding task version, central executive resources, assumed to relate to the temporary maintenance of multi-modal codes in the episodic buffer, appear to underlie the benefit associated with verbalisation.
\end{abstract}

Keywords: visual working memory; visuo-spatial; verbal coding; episodic buffer; central executive; semantic long-term memory. 
The multi-component working memory model comprises two specialised components for the short-term storage of verbal and visuo-spatial material (the phonological loop and visuospatial sketchpad, respectively). A domain-general central executive attentional system supervises and directs the functioning of the specialised components (Baddeley, 2007; Baddeley \& Hitch, 1974). Logie's (1995, 2011; Logie \& van der Meulen, 2009) model further specifies that visuo-spatial working memory is separable, with a short-term visual store specialised for the temporary storage of visual information. While the store is vulnerable to decay and interference, its contents may be refreshed by the active spatial rehearsal mechanism, which can directly draw upon central executive resources. A more recently proposed episodic buffer is specialised for the temporary storage of multi-modal material, and may draw upon long-term memory (Baddeley, 2000; Baddeley, Allen, \& Hitch, 2011; Logie, 2011). However, there is relatively little research investigating the interactions among working memory components, and a paucity of research investigating the influence of verbal coding and semantic knowledge on visual working memory performance.

One example of the way in which memory performance may benefit from processing across multiple modalities is the concreteness effect, by which memory for concrete words (e.g., jacket) is superior to that for abstract words (e.g., jealous). Concrete words are believed to offer increased opportunity for visual elaboration, and are therefore more likely to be recalled from memory due to the dual coding process (Paivio, 1991). Although less research has investigated concreteness in the visual memory domain, verbal coding has been shown to influence visual memory performance. The classic study by Carmichael, Hogan, and Walter (1932) showed that providing verbal labels alongside abstract line drawings influenced how participants reproduced the visual information, inasmuch as the reproductions were less abstract than the original stimulus, and more visually resembled the objects depicted by the 
labels. In this way, concreteness may actually impair recall of an abstract visual stimulus (see also Brandimonte, Hitch, \& Bishop, 1990; Schooler \& Engstler-Schooler, 1990).

However, verbal labels can also enhance visual memory. For example, Bower, Karlin, and Dueck (1975) found that memory for pairs of droodles (very abstract line drawings) was enhanced by labels that not only provided concreteness but also linked the pairs by the same context. More recently, Verhaeghen, Palfai, and Johnson (2006) showed that the presentation of labels alongside to-be-remembered Chinese characters enhances character recognition, and argued that the concreteness (imageability) of the label is key to the enhancement. This supports the idea that establishing a deeper representation, by assimilating stimulus meaning with the surface visual properties, results in superior recall performance (Craik \& Tulving, 1975).

Specifically in the visual working memory domain, Brown, Forbes, and McConnell (2006) showed that some of the abstract stimuli of the Visual Patterns Test (VPT; Della Sala, Gray, Baddeley, Allamano, \& Wilson, 1999; Della Sala, Gray, Baddeley, \& Wilson, 1997), a visual matrix-type task that is designed to be difficult to code verbally, may in fact be amenable to verbal coding (see Figure 1). Based on the ability of individuals to apply verbal labels to the stimuli available from the two parallel versions of the task, Brown et al. selected those patterns that were least amenable to verbal coding for inclusion in a modified version that more tightly restricts task performance to the visual domain. Brown et al. compared the low verbal coding version with performance on the remaining stimuli (i.e., a relatively high verbal coding version), and found that visual working memory capacity was reliably higher in the high verbal coding task. However, it is not yet understood exactly how this systematic difference improves capacity. As visual matrix tasks are well established in the literature for measuring visual working memory, the two task versions offer an ideal opportunity to investigate interactions among working memory components, and between working memory 
and long-term memory. Using a systematic interference paradigm and the low and high verbal coding versions of the VPT, the present experiments represent our initial investigations of these processes.

\section{[Figure 1 about here]}

From the multi-component perspective on working memory, visually presented stimuli may be encoded and stored verbally by the phonological loop, while verbal stimuli may be visually elaborated and stored in the visuo-spatial sketchpad (Baddeley, 2007; Logie, 2011). Higher-order, multi-modal temporary storage may also exist in the episodic buffer, including the use of semantic codes from long-term stored knowledge. However, it is clear from Logie's model that, at initial perception, semantics may be activated rather automatically from our long-term stored knowledge about the visual properties of objects. Theoretically, there are therefore three possibilities for the source of the enhancement related to verbal coding in the VPT: 1) the visual patterns are coded verbally by sub-vocal articulation and stored over the short-term in the phonological store of working memory (Baddeley, 2007; Logie, 2011); 2) the stimuli automatically activate visual semantics, or long-term stored knowledge about the visual properties of objects (Logie, 2011); and 3) drawing on semantic knowledge, multi-modal representations are stored in the episodic buffer, which may draw on central executive resources (Baddeley, 2000; Baddeley et al., 2011; Logie, 2011). In Experiment 1 the potential contribution of the phonological loop to VPT performance was investigated while, in Experiment 2, the involvement of central executive resources was assessed. 


\section{Experiment 1}

Articulatory suppression is a well-established technique for limiting entry of visually presented information into the phonological store of working memory (Baddeley, 2007; see also Larsen \& Baddeley, 2003). If using a visual task that would benefit from verbal recoding, as previously shown with the VPT (Brown et al., 2006), articulatory suppression would be expected to impair performance, by disrupting the verbal coding process. Indeed, Dent and Smyth (2005), who tested visual memory for Japanese Kanji characters that are difficult to label, observed a negative effect of articulatory suppression. Such disruption of visual memory by articulatory suppression would therefore suggest phonological loop recoding and storage otherwise occurs, despite the abstract characteristics of the stimuli. However, as previously stated, it remains possible that there are other sources of the benefit associated with verbalisation of visual material.

Using a visual $n$-back task, Postle, D'Esposito, and Corkin (2005) tested the hypothesis that visual working memory automatically or obligatorily involves semantic coding, even when stimuli are difficult to verbalise. Because task performance was disrupted by the presence of a verbal distractor task during maintenance, Postle et al. concluded that visual working memory automatically recruits semantic knowledge. However, one potential limitation of this work is that the choice of verbal distractor task (semantic categorisation of words) does not allow for disentangling articulation (phonological loop) resources from those involved in retrieval from long-term memory (central executive). A further possibility is that visual images may also have been mentally generated as a result of the presentation of the words, raising the possibility of visual interference. The same limitation may also apply to the fMRI study by Postle and Hamidi (2007), from which the authors concluded that verbal working memory supports visual working memory. Here, the authors stated themselves that semantic support may also be mediating the benefit associated with verbalisation. 
The use of articulatory suppression alongside the two VPT versions may therefore help elucidate the role of verbal elaboration in the VPT, and in visual working memory more generally, by limiting verbal coding and the short-term storage of verbal labels. It is predicted that, if labels are being actively stored in the phonological loop, and verbal working memory is the source of the enhancement, then the difference in performance between the task versions (Brown et al., 2006) would be reduced or even eliminated. However, if the enhancement is operating outside of the phonological loop, at a semantic level, then the difference between the two versions should remain.

One advantage of the present experiment was that individual verbal and visual strategy use was measured, in case the effects of articulatory suppression were mediated by strategy use (e.g., see Logie, Della Sala, Laiacona, Chalmers, \& Wynn, 1996). Individual differences in task approach is a potentially important factor in working memory performance that is rarely considered. For example, while Postle et al. (2005; see also Acheson, MacDonald, \& Postle, 2011) mentioned that participant debriefings were suggestive of verbal coding strategies being used, they did not go as far as to measure strategy use more formally, with a view to allowing such variables to be taken into account during data analysis.

\section{Method}

\section{Participants}

Participants were 40 adults (29 females, 11 males), aged between 18 and 32 years $(M=20.80$, $S D=3.89)$ 


\section{Design}

The experiment took the form of a 2 (control or articulatory suppression; between participants) x 2 (low or high verbal coding version; repeated measures) mixed design. The dependent variable was visual working memory capacity (span).

\section{Materials}

Following Brown et al. (2006) two modified versions of the Visual Patterns Test (Della Sala et al., 1997), that differed in availability of verbal coding, were used. The stimuli were matrix (chequered) patterns consisting of half black and half white cells, ranging in their level of complexity from four (four black cells to be remembered) to fifteen. Each task version comprised three patterns at each level, and therefore a maximum total of 36 experimental trials. One version was low in the availability of verbal coding, while the other was relatively high (see Figure 1). Some of the shapes within the high verbal coding version were canonical, having attracted labels such as letters, numbers, and symbols, while others were amenable to more elaborate labels than this, such as everyday objects and animals. Both task versions were administered via a PC in order to control presentation times precisely (E-Prime 2.0; Psychology Software Tools, Inc.). A brief likert-style questionnaire was also used to allow participants to self-report the extent to which they relied upon visual and verbal strategies (see Appendix 1).

\section{Procedure}

Participants were randomly assigned to the control or articulatory suppression conditions and were administered both versions of the visual working memory task in a counterbalanced order. Standard instructions were provided and each task version was preceded by three practice trials from the same level of complexity as the initial experimental trials (level four). 
Each trial began with presentation of a fixation cross for $1500 \mathrm{~ms}$, pattern display (upon a white background) for $3 \mathrm{~s}$, a maintenance period of $10 \mathrm{~s}$ (during which the screen was black and participants were required to continue looking at the screen), and presentation of the word recall. At recall participants were required to reproduce the pattern on blank paper templates, by placing an $X$ in the cells they remembered as having been black. Each task version terminated when the participant failed to recall correctly at least one trial from a given level. Those participants required to carry out articulatory suppression were instructed to articulate aloud “A, B, C, D, A, B, C, D, A...", and so on, clearly and consistently, at the rate of about two letters per second, from the moment the trial began until recall was complete, in order to limit the possibility of verbal coding even during the latter stages of trials. The experimenter demonstrated the articulatory suppression task and recorded the number of letters articulated during each trial. Mean articulation rate was 30.48 letters per trial $(S D=1.52)$, which did not vary according to task version, $t(19)=-.48, p=.64$. After completing both task versions, participants completed the strategy questionnaire.

\section{Results}

A 2 (interference condition: control, articulatory suppression) x 2 (task version: low, high verbal coding) mixed design Analysis of Variance (ANOVA) was performed on the mean visual working memory span data (see Table 1). This revealed a main effect of task version, $F(1,38)=20.39, M S E=1.35, p<.001, \eta_{p}^{2}=.35$, and a marginal effect of articulatory suppression, $F(1,38)=3.82, M S E=6.63, p=.058, \eta_{p}^{2}=.09$. The interaction was not significant, $F(1,38)=.03, M S E=1.35, p=.88$. 
Reported Strategy. Strategy use data were available for 38 participants. Of note, none of the participants reported exclusive use of a verbal strategy, while 22 of the 38 participants reported either mostly or always using a visual strategy. However, 23 participants reported combining visual and verbal strategies at least sometimes. Interestingly, most participants ( $n$ $=23$ ) rarely or never 'counted up' the black cells, while 32 participants reported attaching verbal labels to the shapes at least sometimes. Spearman correlation analyses revealed relationships between combined strategy use and mean span (collapsed across task version), $r_{s}=-.45, p=.004$, and between attaching verbal labels and mean span, $r_{s}=-.42, p=.009$.

Based on the results of the correlation analyses, to investigate the potential role of strategic approach, a mixed design ANOVA was carried out that took into account the combining variable, which revealed a clear split among the participants. Participants were categorized as combiners (response of always or mostly combined strategies) or noncombiners (response of rarely or never combined strategies), and there were 15 of each group. A 2 (strategic approach; combiner, non-combiner) $\times 2$ (interference condition: control, articulatory suppression) $\times 2$ (task version: low, high verbal coding) mixed design ANOVA was then carried out, which revealed a main effect of task version, $F(1,26)=12.41, M S E=$ $1.18, p=.002, \eta_{\mathrm{p}}^{2}=.32$, a marginal effect of strategic approach, $F(1,26)=4.14, M S E=5.84$, $p=.052, \eta_{\mathrm{p}}^{2}=.14$, but also an interaction between strategic approach and task version, $F(1,26)=4.40, M S E=1.18, p=.046, \eta_{p}^{2}=.15$ (see Figure 2). All other effects and interactions were non-significant (all $p>.28$ ). Bonferroni-corrected paired t-tests revealed that, within the low verbal coding version, the combiners reliably outperformed the noncombiners, $t(28)=3.41, p=.002$, while, within the high verbal coding version, there was no difference according to strategy, $t(28)=1.27, p=.22$. 
Finally, Mann-Whitney U-Tests were carried out to investigate differences in reported strategy use according to presence or absence of articulatory suppression. There was an effect of articulatory suppression on strategy use, $U=104, z=-2.31, p=.02$, with the median response for those in the control condition equally verbal and visual $(M d n=3)$ but that of the articulatory suppression group mostly visual $(M d n=4)$. There was a trend towards articulatory suppression affecting the extent of combined strategy use, $U=120.50, z=-1.79$, $p=.073$, with the median response of the control group lying between mostly and sometimes $(M d n=2.5)$, but that of the articulatory suppression group lying between sometimes and rarely $(M d n=3.5)$. Similarly, there was also a trend towards articulatory suppression affecting the attachment of verbal labels, $U=120, z=-1.84, p=.065$, with the median response of the control group mostly $(M d n=2)$, and that of the articulatory suppression group sometimes $(M d n=3)$. There were no effects of articulatory suppression on the remaining strategy variables (all $p>.65$ ).

\section{Discussion}

The purpose of Experiment 1 was to investigate whether or not the phonological loop is the underlying mechanism responsible for the increased visual working memory capacity previously observed with a version of the VPT that offers greater opportunity for verbal coding (Brown et al., 2006). First, the benefit for the high verbal coding task was replicated (Brown et al., 2006). Second, articulatory suppression successfully limited verbal recoding and rehearsal, as this condition was related to less reported use of a verbal strategy and, marginally, to less reported use of combining visual and verbal strategies. There was also a marginally significant effect of articulatory suppression on visual working memory capacity overall (Delogu, Raffone, \& Olivetti Belardinelli, 2009; Dent \& Smyth, 2005) but, most importantly, it was found not to interact with task version. The phonological loop therefore 
cannot account for the benefit associated with increased availability of verbal coding. Specifically, in the high verbal coding version, participants do not appear to be repeatedly articulating verbal codes any more than in the low verbal coding version.

Reported strategy use, however, was found to influence the results (Logie et al., 1996). In the low verbal coding task condition, those who reported mostly or always combining visual and verbal strategies outperformed those who reported rarely or never combining the strategies, suggesting that central executive resources, for developing and using different strategies, may boost visual working memory performance. Indeed, in a sample of older adults in which visual working memory was assessed using the low verbal coding VPT version, Brown, Brockmole, Gow, and Deary (2012) showed that central executive function specifically related to working with visuo-spatial material (block design) was predictive of performance. However, importantly, the difference between the two strategy groups was absent in the high verbal coding condition. This points to a second source of increased task performance, specifically related to higher verbalisation, which may be the automatic use of semantic knowledge (Mate, Allen, \& Baqués, 2012). The difference between the two task versions may be better understood, then, as the degree of meaning or semantic elaboration (Verhaeghen et al., 2006), rather than the use of verbal coding and articulatory rehearsal per se.

In terms of the responsible mechanism, semantic knowledge may be activated automatically at perception (Logie, 2011), which may be sufficient to allow the high verbal coding version to boost performance. Indeed, the present findings relate to those of Delogu et al. (2009), who showed that, although articulatory suppression interferes with serial recall of easily labeled visual material overall, the superiority associated with bimodal presentation of to-be-remembered items (visual plus auditory stimuli) remains. Delogu et al. concluded that semantic coding mediated the benefit of multi-modal presentations in their study, and that the 
episodic buffer is the candidate interface for these functions (Baddeley et al., 2011; Darling, Allen, Havelka, Campbell, \& Rattray, 2012; Logie 2011). It is possible, however, that episodic buffer storage is an active process drawing on central executive resources. The extent to which the central executive is involved in the high verbal coding version of the VPT was therefore assessed in Experiment 2.

\section{Experiment 2}

The purpose of Experiment 2 was to test the hypothesis that central executive resources are differentially required for the low and high verbal coding versions of the VPT. It was predicted that, if storage in the high verbal coding version relies to a greater extent than the low verbal coding version on generalised resources, central executive suppression during maintenance would reduce or eliminate the benefit. This would suggest that the high verbal coding version involves greater use of semantic codes, assumed to be stored within the multimodal episodic buffer, and that this is an active process (Baddeley, 2000; Baddeley et al., 2011; Delogu et al., 2009; Logie, 2011). Alternatively, if only the automatic activation of semantics is required to produce the advantage associated with the high verbal coding version, and storage of multi-modal codes is cost-free, there should be no differential effect of central executive suppression across the two task versions.

Central executive suppression is typically achieved by the requirement to produce and continuously monitor responding, with the suppression task consistently drawing upon attentional resources. Random generation of material such as letters, words, numbers, or time intervals has therefore been used for this purpose by previous authors (Ang \& Lee, 2010; Baddeley, Emslie, Kolodny, \& Duncan, 1998; Vandierendonck, Kemps, Fastame, \& Szmalec, 2004). Using latent variable analysis, Miyake, Friedman, Emerson, Witzki, and Howerter (2000) showed that random generation of numbers draws upon multiple executive functions, 
specifically, monitoring and updating of working memory representations, and inhibition of automatic, prepotent responses.

In addition to a basic control task, however, it was important to include a spatial interference condition because, in the multiple component model of working memory, the contents of the visual store may be actively refreshed by the spatial rehearsal mechanism, which can draw upon central executive resources (Logie, 2011). In order to be able to disentangle specifically central executive interference from limitations in spatial rehearsal, we included a spatial tapping task for comparison (Darling, Della Sala, \& Logie, 2007; Della Sala et al., 1999). As this was aimed at interfering with visuo-spatial processing, we predicted that this would affect both task versions equally. In order to suppress the central executive, then, we added to the spatial tapping task by requiring that the tapping be conducted at random time intervals. Relative to the basic spatial tapping task, this condition involved central executive suppression only.

\section{Method}

\section{Participants}

The participants were 36 adults, aged 18 to 25 years $(M=20.47, S D=1.25)$, of whom 22 were males. None of the participants had contributed to Experiment 1.

\section{Design}

The experiment took the form of a 2 (low or high verbal coding task version; repeated measures) $\times 3$ (control, spatial interference, or central executive interference; between participants) mixed design. The dependent variable was visual working memory capacity (span). 


\section{Materials}

The working memory task versions were the same as those described in Experiment 1 . In order to carry out the interference tasks, participants were provided with a wooden board, which measured $8 \times 8$ inches and featured four differently shaped markers in each corner.

\section{Procedure}

The procedure was the same as in the control condition of Experiment 1 except that, during the maintenance period of the task, those participants assigned to either the spatial or central executive interference conditions were required to perform spatial tapping. Using their preferred hands, participants tapped in a clockwise direction around the four locations on the wooden board, which was placed on their laps underneath the desk. The experimenter monitored performance to ensure that both tasks were being carried out continuously during the maintenance period, and that the central executive task was carried out randomly. Number of taps per trial was also recorded in both conditions. Strategy use data were unavailable in this experiment.

\section{Results}

The data displayed in Figure 3 were analyzed using a 2 (task version) $x 3$ (interference) mixed ANOVA. There were effects of task version, $F(1,33)=29.93, M S E=.743, p<.001$, $\eta_{\mathrm{p}}^{2}=.48$, and interference, $F(2,33)=83.47, M S E=.932, p<.001, \eta_{\mathrm{p}}^{2}=.84$, but, crucially, also the interaction, $F(2,33)=3.39, M S E=.743, p=.046, \eta_{\mathrm{p}}^{2}=.17$. Planned comparisons showed that the effect of task version was present in both the control, $t(11)=4.37, p=.001$, and spatial interference conditions, $t(11)=3.60, p=.004$, but not in the central executive interference condition, $t(11)=1.24, p=.24$. 


\section{[Figure 3 about here]}

Table 2 shows that tapping performance was the same across the two task versions for both the basic, $t(11)=1.69, p=.12$, and the random spatial tapping task, $t(11)=.38, p=.71$.

\section{[Table 2 about here]}

\section{Discussion}

Experiment 2 was intended to establish the extent to which central executive resources are involved in the low and high verbal coding versions of the VPT. In addition to again replicating the overall benefit of the high verbal coding task version (Brown et al., 2006), and showing overall deficits due to the presence of interference, the interaction effect was observed. Specifically, the enhancement associated with the high verbal coding version appears to have a cognitive cost - central executive resources during maintenance appear to mediate the effect.

It is notable that the spatial interference brought about a significant, sizeable reduction in performance of the VPT. This contrasts with earlier studies showing task-specific visual interference and minimal effects of spatial interference (e.g., Darling et al., 2007; Della Sala et al., 1999). However, this is consistent with research we have previously carried out comparing the effects of visual and spatial interference on the low verbal coding version of the VPT (Brown, McConnell, \& Forbes, 2013; see also Andrade, Kemps, Wernier, May, \& Szmalec, 2002, who also used a visual matrix task). It is therefore possible that short-term visual memory is supported by active spatial refreshment (Logie, 2011) to a greater extent than previously suggested. 


\section{General Discussion}

This study was aimed at investigating the working memory mechanism that underlies the benefit associated with increased availability of verbal coding in the Visual Patterns Test (Brown et al., 2006). This was expected to increase our understanding of interactions among working memory components, and to highlight the circumstances that may lead to higher visual working memory capacity. Theoretically, three candidate mechanisms were potentially responsible for the benefit associated with increased verbalisation: 1) the phonological loop; 2) automatic activation of semantics; and 3) actively stored multi-modal representations in the episodic buffer (Baddeley et al., 2011; Baddeley, 2007; Logie, 2011).

Overall, the findings suggest that the phonological loop is not the source of the benefit associated with higher verbalisation. Rather, while semantic knowledge may be automatically activated at perception, Experiment 2 clearly showed that central executive resources are involved in the resulting working memory capacity increase. This finding is theoretically important, as our ideas regarding the functions required for creating and temporarily storing multi-modal representations have been evolving in recent years, particularly with reference to the involvement of central executive resources.

Baddeley (2000) highlighted that the episodic buffer, while a separate component from the central executive, draws directly upon its active resources. However, more recently, and mainly on the basis of research on visual feature binding, Baddeley et al. (2011) have stated that the attentional resources of the central executive are not required for storage of object representations in the episodic buffer. Logie (2011) also recently theorized that the episodic buffer may store multi-modal representations cost-free in healthy adults, but that cognitive costs come apparent in the damaged brain, for example in Alzheimer's disease patients (Parra et al., 2009). Even within the binding literature, however, there is debate regarding the extent to which attentional resources are necessary to maintain bindings (e.g., Allen et al., 2006; 
Brown \& Brockmole, 2010). Certainly, bound representations appear to be fragile and susceptible to overwriting by the presentation of features similar to those included in target arrays (see Baddeley et al., 2011, for a review), indicating some differentiation from individual feature storage within healthy adults. Certainly, Baddeley et al. did note that there may be other circumstances in which episodic buffer storage is demanding of the central executive. The present study suggests that relating semantic knowledge to the temporary storage of visual information may be one of these circumstances.

It is important to highlight that, although we have focused upon interpreting the findings in relation to the multiple component approach to working memory, which drove the hypotheses and predictions, other models may also be able to account for the data. In particular, there are other influential theories of working memory that emphasise the role of attention in the short-term storage of information (e.g., Barrouillet, Bernardin, \& Camos, 2004; Cowan, 2005; Kane \& Engle, 2002). Cowan's (2001, 2005) model, for example, emphasises that the focus of attention, which draws on central executive processing, allows for the temporary activation of long-term memory traces. This approach could perhaps also account for the increased central executive demand of the task version that incorporates more meaningful information, but would not require a separate episodic buffer component.

Further research is now required to establish more precisely the processes by which multi-modal representations are influenced by central executive functioning. It has been argued that random generation disrupts the ability to monitor and update working memory representations (Miyake et al., 2000). It is therefore possible that the visual representations are actively updated or temporarily bound with semantic constructs from long-term memory, which may be automatically activated initially (Mate et al., 2012). It may also be possible, however, actively to retrieve long-term stored knowledge in relation to the abstract visual representations. Such active semantic elaboration, then, may also be executively demanding 
(Verhaeghen et al., 2006). Furthermore, as the task was performed during only the maintenance period of the memory task in Experiment 2, central executive functions must be necessary during this particular stage of working memory processing. However, it is important to establish whether or not central executive functions are also differentially required during encoding and retrieval in the two versions of the task.

\section{References}

Acheson, D. J., MacDonald, M., C., \& Postle, B. R. (2011). The effect of concurrent semantic categorization on delayed serial recall. Journal of Experimental Psychology: Learning, Memory, and Cognition, 37, 44-59.

Allen, R. J., Baddeley, A. D., \& Hitch, G. J. (2006). Is the binding of visual features in working memory resource-demanding? Journal of Experimental Psychology: General, $135,298-313$.

Andrade, J., Kemps, E., Werniers, Y., May, J., \& Szmalec, A. (2002). Insensitivity of visual short-term memory to irrelevant visual information. The Quarterly Journal of Experimental Psychology, 55A, 753-774.

Ang, S. Y., \& Lee, K. (2010). Exploring developmental differences in visual short-term memory and working memory. Developmental Psychology, 46, 279-285.

Baddeley, A. D. (2000). The episodic buffer: A new component of working memory? Trends in Cognitive Sciences, 4, 417-423.

Baddeley, A. D. (2007). Working memory, Thought and Action. Oxford, UK: Oxford University Press.

Baddeley, A. D., Allen, R. J., \& Hitch, G. H. (2011). Binding in visual working memory: The role of the episodic buffer. Neuropsychologia, 49, 1393-1400. 
Baddeley, A., Emslie, H., Kolodny, J., \& Duncan, J. (1998). Random generation and the executive control of working memory. The Quarterly Journal of Experimental Psychology, 51, 819-852.

Baddeley, A. D., \& Hitch, G. J. (1974). Working memory. In G. H. Bower (Ed.), The psychology of learning and motivation (Vol. 8, pp. 47-90). New York: Academic Press.

Barrouillet, P., Bernardin, S., \& Camos, V. (2004). Time constraints and resource sharing in adults' working memory spans. Journal of Experimental Psychology: General, 133, 83100.

Brandimonte, M. A., Hitch, G. J., \& Bishop, D. V. M. (1992). Influence of short-term memory codes on visual image processing: Evidence from image transformation tasks. Journal of Experimental Psychology: Learning, Memory, and Cognition, 18, 157-165.

Brown, L. A., \& Brockmole, J. R. (2010). The role of attention in binding visual features in working memory: Evidence from cognitive ageing. The Quarterly Journal of Experimental Psychology, 63, 2067-2079.

Bower, G. H., Karlin, M. B., \& Dueck, A. (1975). Comprehension and memory for pictures. Memory \& Cognition, 3, 216-220.

Brown, L. A., Brockmole, J. R., Gow, A. J., \& Deary, I. J. (2012). Processing speed and visuospatial executive function predict visual working memory ability in older adults. Experimental Aging Research, 38, 1-19.

Brown, L. A., Forbes, D., \& McConnell, J. (2006). Limiting the use of verbal coding in the Visual Patterns Test. Quarterly Journal of Experimental Psychology, 59, 1169-1176.

Brown, L. A., McConnell, \& Forbes, D. (2013). Interference and ageing effects in visual working memory. Manuscript in preparation. 
Carmichael, L., Hogan, H. P., \& Walter, A. A. (1932). An experimental study of the effect of language on the reproduction of visually perceived form. Journal of Experimental Psychology, 15, 73-86.

Cowan, N. (2001). The magical number 4 in short-term memory: A reconsideration of mental storage capacity. Behavioral and Brain Sciences, 24, 87-185.

Cowan, N. (2005). Working Memory Capacity. Hove, UK: Psychology Press.

Craik, F. I. M., \& Tulving, E. (1975). Depth of processing and the retention of words in episodic memory. Journal of Experimental Psychology: General, 104, 268-294.

Darling, S., Allen, R. J., Havelka, J., Campbell, A., \& Rattray, E. (2012). Visuospatial bootstrapping: Long-term representations are necessary for implicit binding of verbal and visuospatial working memory. Psychonomic Bulletin \& Review, 19, 258-263.

Darling, S., Della Sala, S., \& Logie, R. H. (2007). Behavioural evidence for separating components within visuo-spatial working memory. Cognitive Processing, 8, 175-181.

Della Sala, S., Gray, C., Baddeley, A., \& Wilson, L. (1997). Visual Patterns Test: a test of short-term visual recall. Bury St. Edmunds, UK: Thames Valley Test Company.

Della Sala, S., Gray, C., Baddeley, A., Allamano, N., \& Wilson, L. (1999). Pattern span: A tool for unwelding visuo-spatial memory. Neuropsychologia, 37, 1189-1199.

Delogu, F., Raffone, A., Olivetti Belardinelli, M. (2009). Semantic encoding in working memory: Is there a (multi)modality effect? Memory, 17, 655-663.

Dent, K., \& Smyth, M. M. (2005). Verbal coding and the storage of form-position associations in visual-spatial short-term memory. Acta Psychologica, 120, 113-140.

Kane, M. J., \& Engle, R. W. (2002). The role of prefrontal cortex in working memory capacity, executive attention, and general fluid intelligence: An individual differences perspective. Psychonomic Bulletin and Review, 9, 637-671. 
Larsen, J. D., \& Baddeley, A. (2003). Disruption of verbal STM by irrelevant speech, articulatory suppression, and manual tapping: Do they have a common source? Quarterly Journal of Experimental Psychology, 56A, 1249-1268.

Logie, R. H. (1995). Visuo-Spatial Working Memory. Hove, UK: Lawrence Erlbaum Associates.

Logie, R. H. (2011). The functional organisation and the capacity limits of working memory. Current Directions in Psychological Science, 20, 240-245.

Logie, R. H., Della Sala, S., Laiacona, M., Chalmers, P., \& Wynn, V. (1996). Group aggregates and individual reliability: The case of verbal short-term memory. Memory \& Cognition, 24, 305-321.

Logie, R. H., \& van der Meulen, M. (2009). Fragmenting and integrating visuo-spatial working memory. In J. R. Brockmole (Ed.), Representing the visual world in memory (pp. 1-32). Hove, UK: Psychology Press.

Mate, J., Allen, R. J., Baqués, J. (2012). What you say matters: Exploring visual-verbal interactions in visual working memory. The Quarterly Journal of Experimental Psychology, 65, 395-400.

Miyake, A., Friedman, N. P., Emerson, M. J., Witzki, A. H., \& Howerter, A. (2000). The unity and diversity of executive functions and their contributions to complex "frontal lobe" tasks: A latent variable analysis. Cognitive Psychology, 41, 49-100.

Paivio, A. (1991). Dual coding theory: retrospect and current status. Canadian Journal of Psychology, 45, 255-287.

Parra, M. A., Abrahams, S., Fabi, K., Logie, R., Luzzi, S., \& Della Sala, S. (2009). Shortterm memory binding deficits in Alzheimer's disease. Brain, 132, 1057-1066. 
Postle, B. R., D’Esposito, M., \& Corkin, S. (2005). Effects of verbal and nonverbal interference on spatial and object visual working memory. Memory \& Cognition, 33, 203-212.

Postle, B. R., \& Hamidi, M. (2007). Nonvisual codes and nonvisual brain areas support visual working memory. Cerebral Cortex, 17, 2151-2162.

Schooler, J. W., \& Engstler-Schooler, T. Y. (1990). Verbal overshadowing of visual memories: Some things are better left unsaid. Cognitive Psychology, 22, 36-71.

Vandierendonck, A., Kemps, E., Fastame, M. C., \& Szmalec, A. (2004). Working memory components of the Corsi blocks task. British Journal of Psychology, 95, 57-79.

Verhaeghen, P., Palfai, T., \& Johnson, M. P. (2006). Verbal labeling as an assimilation mnemonic for abstract visual stimuli: The sample case of recognition memory for Chinese characters. Memory \& Cognition, 34, 795-803. 
Author Notes

We thank Ruth Greenwood-Mears and Sharon McBride for their assistance with data collection in Experiment 1. 


\section{Appendix 1}

1. In this task overall, please rate the extent to which you relied upon a visual and/or verbal strategy to help you remember the checkered patterns. A visual strategy involves concentrating on your mental image of what the pattern looks like. A verbal strategy involves verbalising the features of the pattern and concentrating on that verbal information.

1

"I used a verbal strategy only"
2

"I used mostly verbal but some visual rehearsal"
3

"I used verbal and visual strategies

equally"
4

"I used mostly visual but some verbal rehearsal"

\section{5}

"I used a visual strategy only"

2. To what extent did you combine visual and verbal strategies to help remember individual patterns?

1

"Always"

\section{2}

"Most of the time"
3

"Sometimes"
4

"Rarely"
5

"Never"

3. To what extent did you "count up" the number of black cells?

1

"Always"

\section{2}

"Most of the time"
3

"Sometimes"
4

"Rarely"

\section{5}

"Never"

4. To what extent did you attach verbal labels to some of the individual shapes? (e.g., naming a collection of black cells the letter "L")

\section{1 \\ 2 \\ 3 \\ 4 \\ 5}

"Most of the time"
"Sometimes"
"Rarely"
"Never"

5. To what extent did you focus upon refreshing your mental image of the pattern?

\section{1}

"Always"
2

"Most of the time"
3

"Sometimes"
4

"Rarely"
5

"Never" 
Table 1

Mean visual working memory span (with standard deviations) as a function of articulatory suppression and task version (low or high verbal coding).

Task Version

Low High

Control

$9.28( \pm 2.19)$

$10.50( \pm 1.98)$

Articulatory Suppression

$8.20( \pm 1.83)$

$9.33( \pm 1.98)$ 
Table 2

Mean number of taps per trial (with standard deviations) as a function of interference condition (spatial or random spatial) and task version (low or high verbal coding).

\section{Task Version}

Low High

Spatial Tapping

$33.74( \pm 3.44)$

$33.10( \pm 4.16)$

Random Spatial Tapping

$10.65( \pm 1.39)$

$10.72( \pm 1.45)$ 


\section{Figure Captions}

Figure 1: Sample stimuli from each task version. Stimuli A and B are from Level 8 of the low and high verbal coding task versions, respectively. Stimuli C and D are from Level 11 of the low and high verbal coding task versions, respectively. Stimulus B illustrates some of the more canonical shapes or symbols that may be represent within patterns $[i$, $c$ (back to front)]. Stimulus D illustrates that some verbalisable shapes within patterns may be relatively elaborate (e.g., swan, animal).

Figure 2: Interaction between strategic approach and task version.

Figure 3: Interaction between task version (low or high verbal coding) and interference [control, spatial interference, random spatial (central executive) interference]. 
Figure 1

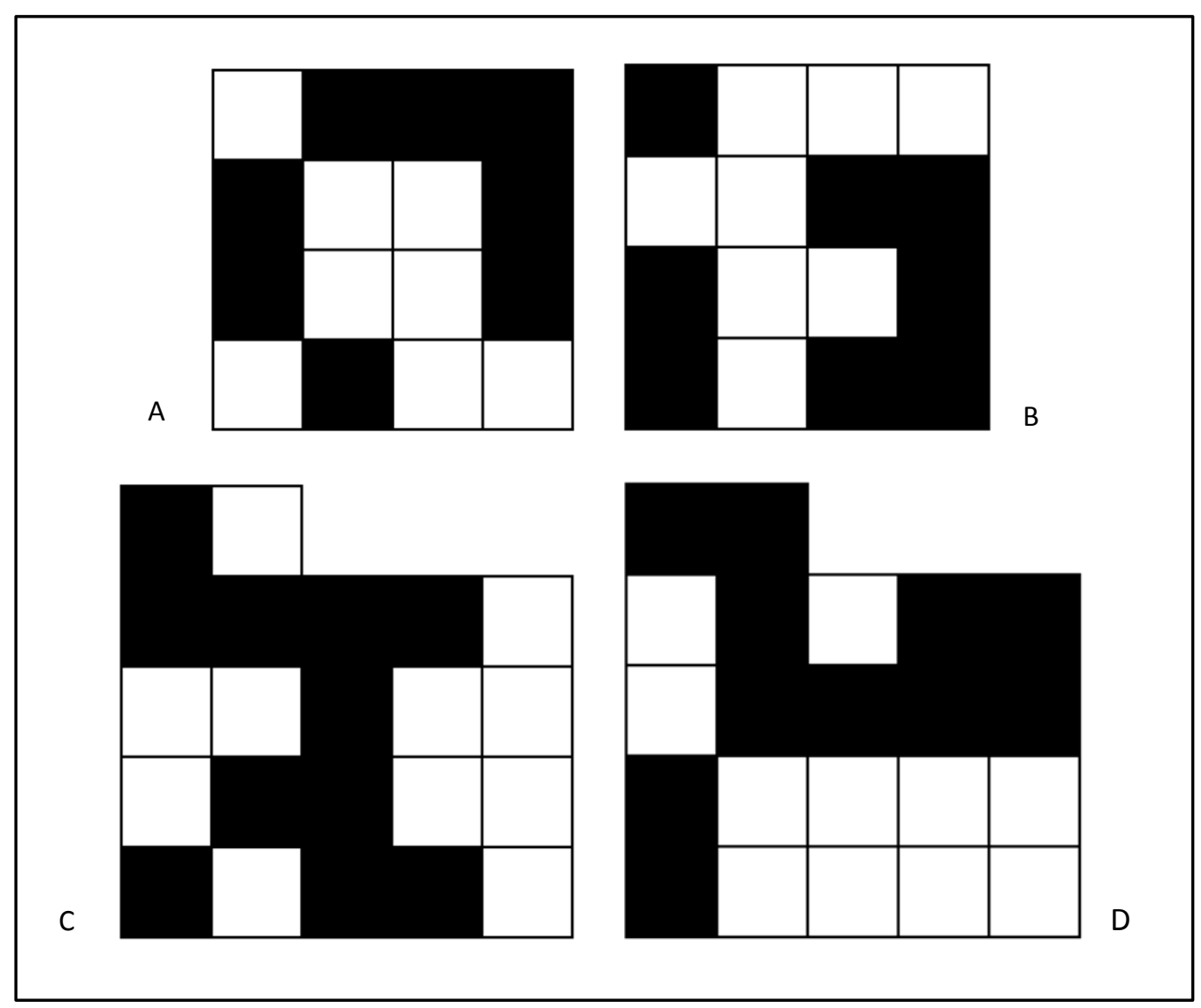


Figure 2

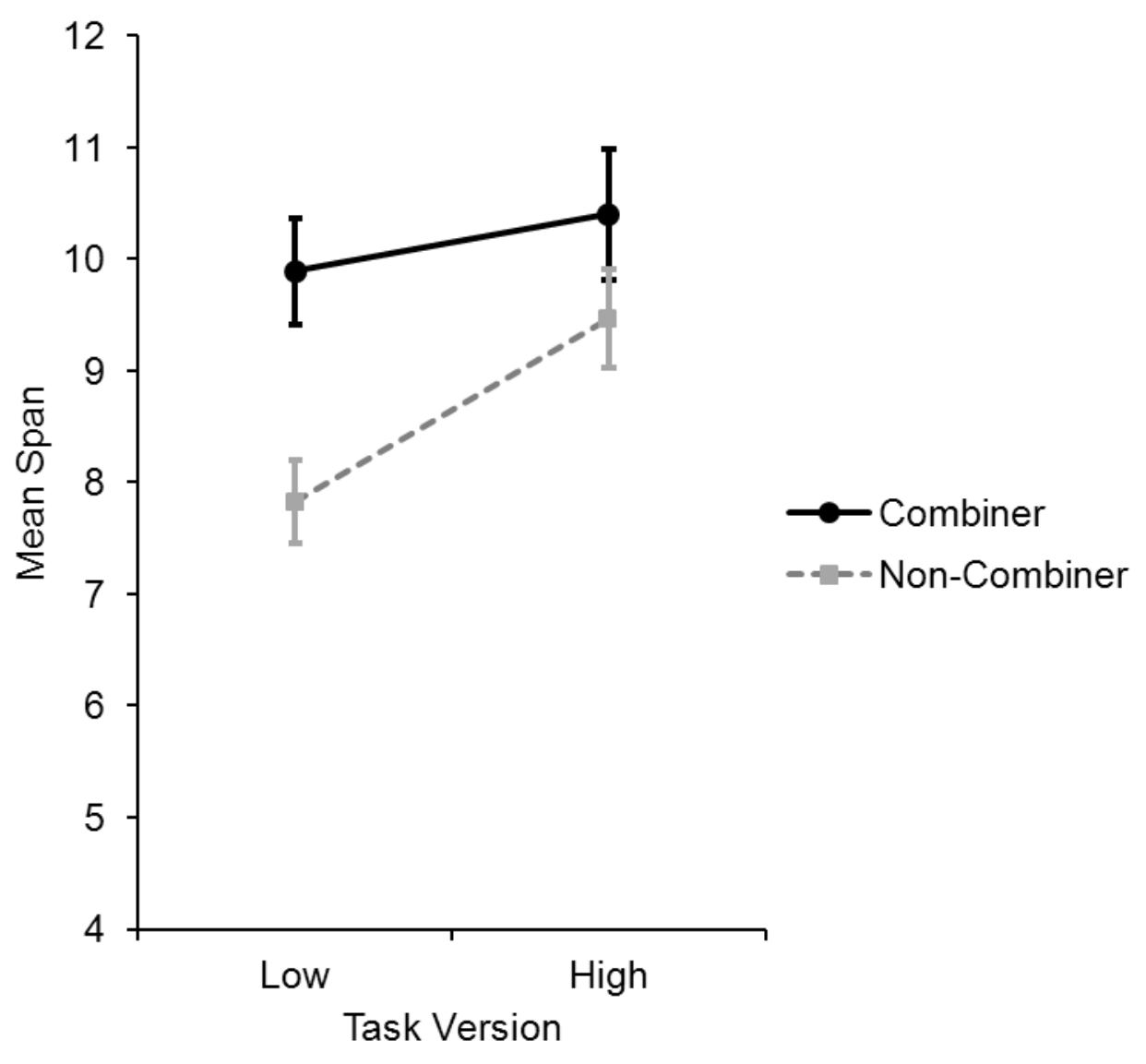


Figure 3

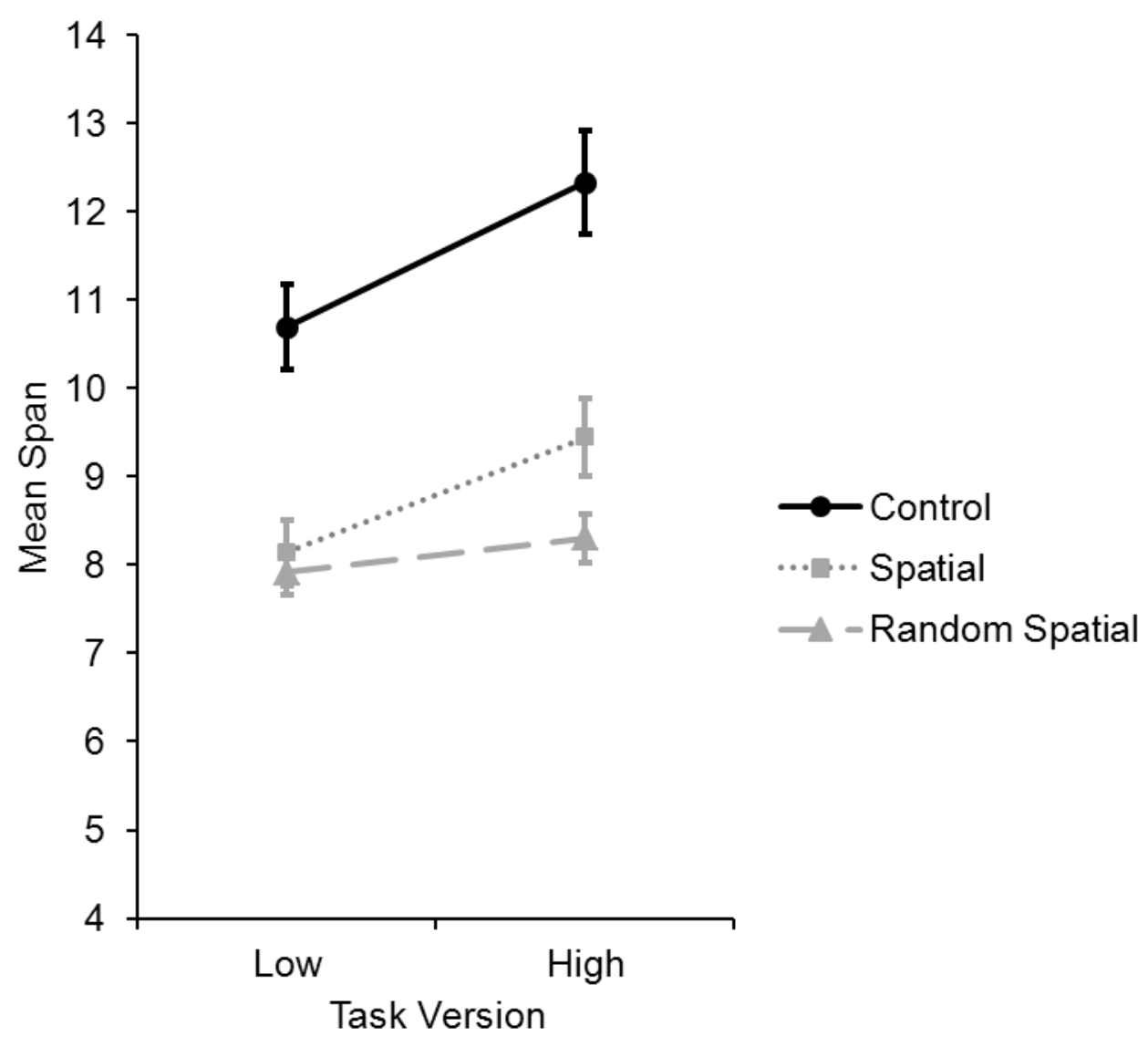

